\title{
The clinical impact of hypoglycemia in hospitalized patients
}

\author{
Vito Borzì, ${ }^{1}$ Andrea Fontanella ${ }^{2}$ \\ ${ }^{1}$ Dipartimento Medicina II, AOU Policlinico-Vittorio Emanuele, Catania; ${ }^{2}$ Dipartimento di Medicina, Ospedale del Buon \\ Consiglio Fatebenefratelli, Napoli, Italy
}

\begin{abstract}
Hypoglycemia is a very common problem, mostly in frail, severely ill, older hospitalized patients. Hypoglycemia has been implicated in the development of adverse clinical outcomes, including an increased mortality. Fear of iatrogenic hypoglycemia remains an obstacle to adequate inpatient glycemic control. It may be considered as a marker of disease, rather than cause in itself of undesirable events. Several factors, such as treatment with exogenous insulin, mismatch between insulin administration and meal intake with the loss of normal counter regulatory responses, place patients with diabetes at higher risk for hypoglycemia than the ones without diabetes. In this review we discuss the causes and predictors of hypoglycemia in non-critically ill hospitalized patients and how to prevent them. Subcutaneous insulin with basal-bolus regimen rather than sliding scale and the use of analogues may minimize hypoglycemic events. In-patient glycemic management should be patient-centered, following the current guidelines, and aimed to prevent hypoglycemic events.
\end{abstract}

\section{Introduction}

The achievement and maintenance of a state of normal blood glucose in hospitalized patients is crucial, owing to the fact that both hypo and hyperglycemia can be extremely dangerous. It has been clearly shown that hyperglycemia is associated with an increase in mortality and in-hospital complications. ${ }^{1}$ Initial small trials have shown that aggressive control of blood glucose with intensive insulin therapy could improve the prognosis of these patients, ${ }^{2,3}$ but subsequent trial, carried out on a larger scale, reported a higher incidence of hypoglycemia in patients in intensive treatment, resulting in a worse prognosis. ${ }^{4}$ This evidence resulted in defining protocols of treatment of hyperglycemia in hospitalized patients, in which a reasonable and acceptable glycemic control is defined as a blood glucose varying between

Correspondence: Andrea Fontanella, Dipartimento di Medicina, Ospedale del Buon Consiglio Fatebenefratelli, via Manzoni 220, 80123 Napoli, Italy.

E-mail: andreafontanella52@gmail.com

Key words: hypoglycemia, diabetes, non-critical illness, inpatients.

Received for publication: 6 October 2014.

Accepted for publication: 3 December 2014.

This work is licensed under a Creative Commons Attribution NonCommercial 3.0 License (CC BY-NC 3.0).

CC Copyright V. Borzi and A. Fontanella, 2015

Licensee PAGEPress, Italy

Italian Journal of Medicine 2015; 9:11-19

doi:10.4081/itjm.2015.549
99 and $180 \mathrm{mg} / \mathrm{dL}$ for the most part of patients. ${ }^{5}$ If we take into account that in the United States in 2010 were discharged approximately 8.3 million people with a diagnosis of diabetes, i.e. $21.3 \%$ of all patients discharged, ${ }^{6}$ we understand the extent of the problem and its clinical importance. Hypoglycemia causes acute events, potentially fatal; therefore it remains a major obstacle to the optimal glycemic treatment in the hospitalized patient. It is now well understood that patients with both spontaneous and iatrogenic hypoglycemia are at a high risk of complications and mortality, as well as of a greater length of hospital stay. Although this association is now well-established, yet it is not clearly documented a direct causality. In this review we will try to examine the causes of hypoglycemia in patients hospitalized in the Internal Medicine Departments and the importance of preventing such events by means of appropriate therapeutic strategies.

\section{Definition and prevalence of hypoglycemia}

A blood sugar level below $70 \mathrm{mg} / \mathrm{dL}$ is the threshold generally used to indicate hypoglycemia. The true hypoglycemia is defined by the activation of counter-regulatory mechanisms and this varies from individual to individual. The release of epinephrine and glucagon occurs at blood glucose levels close to $70 \mathrm{mg} / \mathrm{dL}$, followed by the release of somatotropin and cortisol when the blood sugar level goes down to $60 \mathrm{mg} / \mathrm{dL}$. The onset of autonomic symptoms requires lower blood glucose levels, usually around $50 \mathrm{mg} / \mathrm{dL}{ }^{7}$ It has been defined by the term severe hypoglycemia low blood sugar lower than $50 \mathrm{mg} / \mathrm{dL}$ or requiring the assistance of another person. Using in patients hospitalized the counter regulation thresholds, derived from studies conducted in 
outpatients, could be problematic, as the former may have an impaired counter-regulation. ${ }^{89}$ In addition, hospital patients may exhibit only neuroglycopenic signs, without any preceding autonomic symptoms. This is due to many factors including the polypharmacy, multiorgan dysfunction and the presence of long lasting diabetes, all factors usually present in the internal medicine patient. ${ }^{10}$ Therefore, to define a hypoglycemia in hospitalized patients, rather than using the glucose counter-regulation thresholds or the development of symptoms, it is recommended to safely cut off $<70$ $\mathrm{mg} / \mathrm{dL}$, a value recently adopted by two major American scientific societies. ${ }^{11}$

Owing to variations in defining of hypoglycemia, it is not surprising that the estimation of its prevalence in hospitalized patients shows large fluctuations, ranging from $3 \%$ to $30 \%$. Many studies, moreover, do not distinguish between patients with and without diabetes, nor among those critically ill - admitted in Intensive Care Units (ICU) compared to not critical patients. Among the people with and without diabetes admitted to an internal medicine ward, the incidence of hypoglycemia (defined as blood glucose $<70$ $\mathrm{mg} / \mathrm{dL}$ ) varies between $3.5 \%$ and $10.5 \% .{ }^{12}$ Just in patients with diabetes, however, hypoglycemia may occur in $12 \%-18 \%$ of cases, or highest percentage if treated with more aggressive glucose-lowering therapy. ${ }^{13}$ In a retrospective review, among more than 4000 admissions in Internal Medicine Department, hypoglycemia was found in $8 \%$ of hospitalizations and in $3 \%$ of patients admitted. ${ }^{14}$ In a study including data from 575 hospitals, hypoglycemia (blood glucose $<70$ $\mathrm{mg} / \mathrm{dL}$ ) occurred in $6.3 \%$ of patients admitted to the ICU and in $5.7 \%$ of patients in normal wards. ${ }^{15}$ In several intervention studies performed on patients admitted in ICU higher incidences of hypoglycemia were observed, with values ranging from $5 \%$ to $18 \%$ in the intensive treatment group. ${ }^{3,4,16}$

\section{Risk factors of hypoglycemia}

Factors contributing to hypoglycemic events in hospitalized patient may be multiple (Table 1). Although diabetes and the subsequent use of hypoglycemic drugs is the most common risk factor, only $50 \%$ of hypoglycemic events occurs in diabetic patients or in those receiving insulin. ${ }^{17,18}$ The spontaneous hypoglycemia, which occurs without any clear precipitating cause, must be distinguished from that one drug-induced, even as it has a poor prognosis and a different pathogenesis. Risk factors for spontaneous hypoglycemia are septic shock, comorbidities (as defined by the Charlson comorbidity index), drug interactions in polypharmacy, renal failure, liver or adrenal disease, severe heart failure and neoplasms. ${ }^{17,18} \mathrm{We}$ have always to remember that during hospitalization, patients undergo changes of their insulin sensitivity and counter-regulatory hormone, secondary to the disease, procedures and treatment carried out. ${ }^{19,20}$ Older patients also have often organ dysfunction age related, which alters insulin sensitivity and drug metabolism, resulting in the enhancement of hypoglycemic events. ${ }^{10}$ Elderly people show symptoms of hypoglycemia at a considerably lower glycemic threshold compared to the young $(54+3.6 \mathrm{mg} / \mathrm{dL}$ vs $65+1.8$ $\mathrm{mg} / \mathrm{dL})^{9}$ and frequently for their altered mental conditions are not able to communicate their symptoms. In diabetic patients, the most obvious risk factor for hypoglycemia is the exogenous administration of insulin or the stimulation of insulin secretion by secretagogues containing drugs. In these cases, since that hyperinsulinemia is drug-induced and non-physiological, the levels of insulin will not reduce in response to the lowering of blood sugar. You have also to take into account that the hormonal counter-regulatory response to hypoglycemia is often impaired in patients with diabetes (especially of type 1 and with long duration of diabetes), which have a reduced secretory response to glucagon and epinephrine. Another important factor to consider is that previous recurrent hypoglycemias facilitate further hypoglycemic episodes, since the threshold for counter-regulatory response increases when blood sugar levels are high, while it decreases in the case of normal values..$^{21}$ Therefore, a single episode of hypoglycemia increases the risk of subsequent episodes, thus triggering a vicious cycle of recurrent hypoglycemia. This phenomenon is well documented on the inpatient treatment. One study reported that $40 \%$ of hospitalized patients had recurrent episodes of hypoglycemia, ${ }^{22}$ while in another study severe hypoglycemic episodes were preceded by mild hypoglycemia in more than $60 \%$ of cases. ${ }^{23}$ Another factor favoring a dysregulation of the counter is the

Table 1. Risk factors for hypoglycemia in hospitalized patients.

Iatrogenic

- Too aggressive treatment protocols

- Poor coordination between the temporal administration of insulin and meals

- Unscheduled suspension in eating

- Interruption of steroid therapy without relating changes in insulin

- Using the sliding scale

Organic

- History of frequent hypoglycemia or unawareness hypoglycemia

- Autonomic neuropathy

- Advanced age with or without cognitive impairment

- Sepsis

- Critical illness

- Renal or hepatic impairment

- Cancer

- Adrenal insufficiency 
presence of autonomic neuropathy. ${ }^{24}$ Although there are no studies in hospitalized patients, it is very likely that the diabetic autonomic neuropathy may be a significant risk factor for hypoglycemia in diabetic inpatients. Insulin therapy is elective in the hospital for blood glucose control. Although its pharmacokinetic profile is very well predictable with respect to non-insulin therapies and its dosage can be very flexible, insulin is one of the high-alert medications in hospitalized patients. ${ }^{25}$ The association between hypoglycemia and insulin administration is dose dependent. A retrospective case-control study of 1990 patients with diabetes admitted to hospital showed that the risk of hypoglycemia was doubling with insulin doses between 0.6 and $0.8 \mathrm{U} / \mathrm{kg} / \mathrm{day}$, while threefold with insulin doses $>0.8 \mathrm{U} / \mathrm{kg} / \mathrm{day}$; on the other hand there was no increase in hypoglycemic events at doses of 0.2$0.6 \mathrm{U} / \mathrm{kg} /$ day, compared to doses $<0.2 \mathrm{U} / \mathrm{kg} /$ day. ${ }^{26}$ The failure to correct insulin doses in the case of reduced food intake contributes to the increased risk of hypoglycemia. ${ }^{27}$ The sudden interruption of enteral or parenteral nutrition is yet another important risk factor, unless another source of glucose is not administered. ${ }^{28}$ Any variation of the type of nutrition, without an adequate proportionate change of the doses of insulin, has been shown to be responsible for $82 \%$ of hypoglycemic events. ${ }^{19}$ Patients who are regularly and at the right times fed may be at risk of hypoglycemia too, without adequate pre-prandial blood glucose monitoring and timing of insulin administration and/or meals control $;{ }^{29}$ finally, changes in the content of carbohydrates in the meal is another risk factor in subjects receiving fixed insulin doses with meals. ${ }^{27}$ Despite the scientific evidence proved the ineffectiveness and the lack of security of sliding scale insulin (SSI), this is unfortunately still a very common practice in hospital wards. ${ }^{30}$ The SSI refers to the practice of administering doses of regular insulin or rapid analogous, depending on blood glucose values at the time. There is a large variability between professionals in establishing at which blood glucose levels to start the SSI and what doses to use. ${ }^{31}$ This regimen is usually taken before meals and at bedtime, without taking into consideration if the patient could or not ingest calories at bedtime. Therefore, the use of SSI contributes substantially to hypoglycemias, especially at night. The SSI should instead be understood as a correction or additional insulin in patients who are already running a previously established basal-bolus dose. In these situations, small doses of rapid-acting insulin are administered before meals (in patients who are fed) or every $6 \mathrm{~h}$ (in patients who do not eat or in artificial nutrition). Sudden changes in the normal hospital routine, such as the abrupt execution of the procedures that force the patient to skip a meal, ${ }^{19}$ the unpredictability of the dietary intake, the interruption of steroid treatment without any changes of hypoglycemic therapy, are all factors contributing to the onset of hypoglycemia. Finally, even in diabetic patients, all those risk factors for spontaneous hypoglycemia listed above, in addition to the risks of insulin therapy, may contribute to the high incidence of hypoglycemic events.

\section{Consequences of hypoglycemia}

The consequences of the acute hypoglycemia concern mainly the pro-thrombotic and pro-inflammatory effects. Indeed, in experimental models, hypoglycemia causes an increase in catecholamine levels, inflammatory markers, platelet activation and endothelial dysfunction..$^{32,33}$ Both in healthy subjects and type 1 diabetes, hypoglycemia results in an increase of monocytes adhesiveness, markers of platelet activation (Pselectin) and inflammation (monocytes CD-40, IL-6 and hrCRP), and of adhesion molecules (VCAM-1, ICAM-1, E-selectin). ${ }^{32,33}$ Some of the pro-thrombotic and pro-inflammatory effects of acute hypoglycemia may be reduced by $\beta$-blockers, thereby assuming that these changes are induced through adrenergic activation and therefore that they can be attenuated in individuals with an impaired counter-regulation. ${ }^{34}$ There are still no studies demonstrating whether acute hypoglycemia effects may also include patients who, already sick and hospitalized, show in themselves an increase of inflammatory mediators, catecholamines and stress hormones. Hypoglycemia can lead to cardiac complications, especially ischemia and arrhythmias, that were even associated with mortality. ${ }^{35}$ Hypoglycemia has been associated with transient electrocardiogram changes during a continuous blood glucose and electrocardiographic monitoring. ${ }^{35}$ and to the QT interval lengthening, which may trigger reentrant arrhythmias. ${ }^{36,37}$ These cardiac abnormalities might be responsible for the dead in bed syndrome, i.e. the phenomenon of the sudden death at night, described in young patients with type 1 diabetes. Furthermore, patients with long-term diabetes can develop a cardiovascular autonomic neuropathy, a chronic condition associated with prolongation of the QT interval and an increased risk of sudden death. ${ }^{38} \mathrm{To}$ date, there are no data demonstrating these cardiovascular alterations in hospitalized patients, since the existing literature relates only to outpatients. There is still a lot of debate on whether hypoglycemia in itself may be the cause of increased cardiovascular events. Indeed, large trials performed in hospitalized patients treated with intensive insulin therapy have reported rare acute cardiac complications, despite frequent and severe hypoglycemias. ${ }^{39}$ In the DIGAMI 1 study, in which all patients were hospitalized for acute myocardial infarction (AMI), the arm in intensive insulin therapy had the same incidence of re-infarction, ventricular fibrillation 
and atrio-ventricular conduction disorders, compared to the control arm, despite the fact that $15 \%$ of them presented hypoglycemias $v s$ the control group, who had not hypoglycemic events. ${ }^{40}$ Even the EURODIAB Prospective Complications Trial ${ }^{41}$ showed that subjects treated in an intensive way, and thus with a higher incidence of hypoglycemia, did not have an increase in cardiovascular events or of inflammation markers. The lower incidence of hypoglycemia-related cardiac morbidity observed in the trials could be due to the strict patient monitoring, resulting in a short duration of the hypoglycemia. In addition, during hospitalization, the highest concentration of circulating catecholamines, as well as the administration of inotropic drugs, may attenuate the heart rate response to hypoglycemia. Evidence that the daily clinical practice could be quite different, can be inferred from a recent study, carried out by questionnaire, showed how hypoglycemia in hospitalized patients can lead to higher numbers of adverse events, compared to what was so far shown, whereas this is the case for hypoglycemic episodes following the treatment of hyperkalemia ${ }^{42}$ Hypoglycemia may also cause a variety of neurologic manifestations, ranging from simple irritability to coma. However, cerebral death is very rare and requires a prolonged and severe hypoglycemia. In primates the insulin-induced hypoglycemia can cause brain death when the blood sugar is still below $20 \mathrm{mg} / \mathrm{dL}$ for at least $5 \mathrm{~h} \cdot{ }^{43}$ Human studies are limited and the best evidence comes from the DCCT/EDIC trial: a careful monitoring of patients in the intensive treatment arm showed that, although the incidence of repeated and severe hypoglycemic episodes, there was no evidence of brain damage. ${ }^{44}$ In patients hospitalized with severe iatrogenic hypoglycemic episodes, hypoglycemia did not only hesitate in neurological deficits, but, on the contrary, was associated with favorable outcomes. ${ }^{2}$ In conclusion, brain death from hypoglycemia, although possible, is very rare in clinical practice. Several studies on hospitalized patients have shown a correlation between hypoglycemia and mortality, ${ }^{14,39,45}$ although the direct liability still remains very uncertain. An observational study proved a kind of J-curve between blood glucose levels and the likelihood of mortality, with lower fatalities with blood glucose levels ranging from 100 to 150 $\mathrm{mg} / \mathrm{dL} .{ }^{46}$ This associated increased mortality in hospitalized patients may not be caused by hypoglycemia in itself, but may instead be due to the association between hypoglycemic episodes and comorbidities, where hypoglycemic episodes could be a marker of frailty. In hospitalized patients spontaneous hypoglycemias occur without clear triggering factors, but they are associated with higher mortality compared to iatrogenic hypoglycemias. A retrospective study of a large cohort of patients with or without diabetes, admitted to Departments of Internal Medicine, showed that mortality was higher among patients who had spontaneous hypoglycemias. ${ }^{17}$ After adjustment for comorbidities, hypoglycemia was no longer an independent risk factor for mortality, thereby reinforcing the concept that hypoglycemia acts as a marker of severe underlying disease, rather than as a direct cause of death. Similar results were found in patients with AMI, about $50 \%$ of them diabetics. ${ }^{47} \mathrm{~A}$ recent case-control study about hospitalized patients reported similar data, even showing that hypoglycemia, either spontaneous or iatrogenic, occurred in greater number among the most vulnerable patients. ${ }^{45}$

The NICE-SUGAR study showed that mortality attributable to hypoglycemia was related to its severity and duration, with no distinction between the type (intensive or conventional) of treatment and the presence (or not) of diabetes. ${ }^{39}$ In conclusion, we could say that spontaneous hypoglycemia is associated with a worse prognosis, while hypoglycemic therapy may unmask a propensity to develop hypoglycemia in hospitalized patients with severe comorbidities. In addition to mortality rate, hypoglycemia in hospitalized patients results in an increase in hospital stays and increased costs. A study on more than 100,000 hospitalized patients with diabetes highlighted that patients whose average blood glucose was $<70 \mathrm{mg} / \mathrm{dL}$ had a length of stay higher than three days, in comparison to those who not had low blood glucose levels, and hypoglycemia linked to about $39 \%$ increase in hospital admissions..$^{48}$ In another study of 2582 diabetic patients admitted to Internal Medicine Wards, it has been shown as for every day in which the blood sugar was maintained lower than $50 \mathrm{mg} / \mathrm{dL}$, the length of hospital stay increased approximately about by 2.5 days. ${ }^{14}$

\section{Preventing hypoglycemia}

Although the fear of hypoglycemia constitutes a barrier to the optimal glycemic control, subsequent inappropriate treatment of hyperglycemia would be an undesirable approach. ${ }^{49}$ Thus, prevention of hypoglycemic episodes should be achieved through a $p a-$ tient-centered process, able to share appropriate treatment upon the specific nutritional needs of the patient, with proper glucose monitoring, appropriate hospital procedures, and, above all, the capacity to adapt treatment and care, according to the actual clinical needs of our patients (Table 2). The subcutaneous insulin therapy, in accordance to the basal-bolus approach, involves a scheduled administration of therapy, with the long acting insulin approximately represented as $50 \%$ of the total daily dose, and the rest administered as rapid-acting insulin, before the three main meals, 20$30 \%$ at breakfast, lunch and dinner $30-40 \%$.

Many steps of schemes correction of insulin therapy have been formulated, but the most practical approach seems to be the rule of 1500 , for estimating the 
factor of insulin sensitivity. Established the value of insulin sensitivity factor, on the basis of blood glucose data, we can calculate the doses of insulin to administer as a correction factor ${ }^{50}$ (Table 3 ). Thus, the use of this therapeutic approach allows combining doses of insulin-to-insulin sensitivity of the patient, therefore by treating hyperglycemia without any major risk of hypoglycemia. Regarding the calculation of the total dose of insulin in administering the basal-bolus pattern, this varies from 0.2-0.6 U/kg/day, always taking into account the clinical state of the patient. The lower dose $(0.2 \mathrm{U} / \mathrm{kg} /$ day $)$ may be used to slim patients, or in chronic renal insufficiency or in seniors (older than 70 years); the higher dose can be used in overweight people with acute illnesses (increased insulin resistance) or who are receiving steroids. Of course, the doses of insulin should be amended on the basis of the daily blood glucose monitoring. ${ }^{5}$ It is very important to identify patients at risk of hypoglycemic episodes: in these subjects is necessary to implement systems of care by more frequent clinical monitoring, in order to minimize the frequency and the severity of such episodes. Patients with chronic renal failure are a group at high risk for hypoglycemic events. In a study of inpatients with glomerular filtration rate lower than $45 \mathrm{~mL} / \mathrm{min}$ randomized to a basal-bolus therapy by glargine and glulisine, using two strengths ( 0.25 or $0.50 \mathrm{U} / \mathrm{kg} /$ day), the lower dose group had a slight, but not significant, lower incidence of hypoglycemic episodes compared to the group with the highest dose ( $15.8 \%$ vs $30.0 \%, \mathrm{P}=0.08)$, but with no increase in incidence of hyperglycemia. Also in the arm at lower administered doses, hypoglycemic events were present, this strengthening the need for a tight control of blood glucose levels and insulin doses in patients at higher risk of hypoglycemic episodes. ${ }^{51}$ For many years there have been developed fast and basal analogues, able, for their own pharmacokinetic profiles, to provide better efficacy and safety. The rapid acting analogues (aspart, lispro or glulisine) lead to some substantial benefits, compared to regular insulin, when used to control postprandial blood glucose values. While regular insulin needs to be injected 30' before the meals, the rapid acting analogues can be administered immediately before or after a meal, without any change in their action. ${ }^{52,53}$ This is a great advantage in the management of blood glucose levels in the hospital, where often it is not possible to accurately deter-

Table 2. Prevention of hypoglycemia.

- Avoid using oral hypoglycemic agents

- Carefully consider the clinical status and any risk factors in your patient

- Avoid the use of premixed insulin that do not give a physiological replacement therapy

- Use the analogues, preferably the rapid and basal ones

- Use the correct dose, according to the patient's sensitivity

- If food intake is not predictable, administer the similar rapid at mid-meal

- Improve blood glucose monitoring

- For patients at higher risk of hypoglycemia use higher glycemic targets

- Implement the use of guidelines and protocols

- Raise $\beta$ and train in your medical and nursing staff

Table 3. The calculation of correction bolus based on insulin sensitivity.

\begin{tabular}{|c|c|c|c|c|}
\hline Blood glucose (mg/dL) & Scale 1 & Scale 2 & Scale 3 & Scale 4 \\
\hline $151-200$ & 1 & 2 & 3 & 4 \\
\hline $201-250$ & 2 & 4 & 6 & 8 \\
\hline $251-300$ & 3 & 6 & 9 & 12 \\
\hline $301-350$ & 4 & 8 & 12 & 16 \\
\hline $351-400$ & 5 & 10 & 15 & 20 \\
\hline Insulin sensitivity (ISF)* & 50 & 25 & 16.7 & 12.5 \\
\hline
\end{tabular}

*Insulin sensitivity factor (ISF) is the decrease in blood glucose following administration of $1 \mathrm{U}$ of insulin. ISF is calculated by the rule 1500 (ISF=1500 / TDD), where TDD stands for the total daily dose of insulin (rapid+basal). 
mine the time or the amount of food that the patient will take. In addition, the peak effect is achieved earlier than with regular insulin, resulting in improved control of postprandial blood glucose, while their shortest duration of action reduces the risk of hypoglycemic episodes later or pre meal. ${ }^{54-56}$ Several clinical trials have shown that the use of rapid acting analogues results in a lower incidence of hypoglycemic events, with the same glycemic control. ${ }^{57-59}$ The physiological basal insulin secretion is mimicked in our diabetic patients by the use of basal or late insulin. The ideal basal insulin should have a 24-h action duration, in absence of peaks. Unfortunately the neutral protamine Hagedorn (NPH) insulin does not absolutely mimic the physiological secretion, since its single administration does not cover the $24 \mathrm{~h}$ and its action has a peak toward the second to fourth hour after its administration. These features affect significantly the blood glucose, resulting in potential nocturnal hypoglycemias and hyperglycemia upon awakening. The long-acting basal analogues (glargine and detemir) have solved these problems in a very satisfactory way. Their long duration and their flat profile allow the achievement of good metabolic control, with a significant reduction of night hypoglycemic episodes especially compared to NPH insulin. ${ }^{60,61}$ Basal analogues show even less pharmacokinetic variability, resulting in greater stability blood glucose inter and intra-individual compared to NPH. ${ }^{62}$ According to these considerations, the American Association of Clinical Endocrinologists/American College of Endocrinology (AACE/ACE)'s statements strongly recommended the use of analogues (glargine and detemir) compared to NPH as insulin baseline. ${ }^{63}$ The FADOI-DIAMOND study sub-analysis showed that among diabetic patients admitted to Italian Internal Medicine Units, patients treated with a basal-bolus scheme with analogues have less hypoglycemic episodes compared to those treated with an insulin alone or with a basal sliding scale. In this study was confirmed that hypoglycemia may worse hospital indicators, by a longer hospital stay $(12.7 \pm 10.9 \mathrm{vs}$ $9.6 \pm 6.5$ days, $\mathrm{P}<0.01) .{ }^{64} \mathrm{In}$ addition, in order to facilitate an adequate glycemic control with the lower possible risk of hypoglycemia, further progress in the field of research of insulin treatment have been defined, by the near future availability, in Italy too, of degludec insulin. This is a new ultra-longacting basal insulin (half-life: about $25 \mathrm{~h}$ ), which forms soluble multi-hexamers, when injected subcutaneously, determining a deposit from where the degludec insulin is absorbed continuously and slowly in the circulation, leading to a uniform and stable hypoglycemic effect. Degludec insulin shows an intra-patient variability from day to day hypoglycemic effect four times lower in terms of coefficients of variation compared to in- sulin glargine ${ }^{65}$ Pivotal trials by this new type of insulin showed that in type 1 diabetes, degludec insulin, compared with glargine in a basal-bolus regimen with a rapid acting analogues, with the same glycemic control, resulted in less nocturnal hypoglycemia. ${ }^{66}$ In type 2 diabetes, when compared to the glargine in a basal dose regimen only on equal glycemic control, it showed a significant reduction of all $24 \mathrm{~h}$-daily, not only nocturnal, hypoglycemic episodes. ${ }^{67}$ These data were well confirmed in elderly subjects too, those aged over 65 years ${ }^{68}$ normally more vulnerable to hypoglycemic episodes. In addition, more than $24 \mathrm{~h}$ halflife of this new insulin allows its administration not directly time-dependent, increasing the flexibility of insulin therapy, essential in the management of hyperglycemia in hospitalized patients.

One study ${ }^{69}$ evaluated patients with type 2 diabetes, who were divided into three groups: two groups were receiving insulin glargine or degludec (IDeg) every $24 \mathrm{~h}$, while the third received degludec at different times, with a variation in the last administration that ranging from 8 to $40 \mathrm{~h}$; however, over the week, patients always received seven insulin injections, as well as other groups. This study was designed to explore from the point of view of safety and efficacy, the impact of the variability of basal insulin delivery. Results showed that patients in which degludec insulin was administered at variable intervals, at the end of the 26 weeks of treatment reached the same glycemic control, without any increasing risk of hypoglycemia, compared to those in whom the same deglutec or glargine were administered at fixed times. The use of extreme dosing intervals of 8-40 h demonstrates that the daily injection time of IDeg can be varied without compromising glycemic control or safety.

One last, but not least important factor in the prevention of hypoglycemic episodes, is a careful analysis of hospital processes and continuous updating and review of the know how in both medical and nursing staff. The recent study on FADOI-DIAMOND has shown how an educational program involves a continuing improvement in the intra-hospital treatment, resulting in a significant increase in the efficacy and safety of insulin therapy. ${ }^{70}$

\section{Conclusions}

Hypoglycemia is a very common problem among hospitalized patients with or without diabetes, associated with higher mortality. Hypoglycemia, related to severe disease and comorbidities, may suggest it as is one marker, rather than a direct cause, of increased risk of mortality. In hospital, mostly in Internal Medicine Wards, it is very important to take all appropriate precautions to avoid hypoglycemic events that might cause adverse outcomes. Fortunately, hypoglycemia is pre- 
dictable and preventable if particular attention is given to trigger factors, by overall clinical assessment of patients and the choice of the best treatment options, with implementation of the best specific protocols.

\section{References}

1. Umpierrez GE, Isaacs SD, Bazargan N, et al. Hyperglycemia: an independent marker of in-hospital mortality inpatients with undiagnosed diabetes. J Clin Endocrinol Metab 2002;87:978-82.

2. Van den Berghe G, Wouters P, Weekers F, et al. Intensive insulin therapy in critically ill patients. New Engl J Med 2001;345:1359-67.

3. Van den Berghe G, Wilmer A, Hermans G, et al. Intensive insulin therapy in the medical ICU. New Engl J Med 2006;354:449-61.

4. NICE-SUGAR Study Investigators; Finfer S, Chittock $\mathrm{DR}$, et al. Intensive versus conventional glucose control in critically ill patients. New Engl J Med 2009;360: 1283-97.

5. Umpierrez G, Hellman R, Korytkowski MT, et al. Management ofhyperglycemia in hospitalized patients in noncritical care setting: anendocrine society clinical practice guideline. J Clin Endocrinol Metab 2012;97:16-38.

6. Young I, Sicbodnok M, Sands L. Bar code technology and medication administration error. J Patient Saf 2010; 6:115-20.

7. Schwartz NS, Clutter WE, Shah SD, Cryer PE. Glycemic thresholdsfor activation of glucose counterregulatory systems are higherthan the threshold for symptoms. J Clin Inv 1987;79:777-81.

8. Dagogo-Jack SE, Craft S, Cryer PE. Hypoglycemia-associated autonomic failure in insulin-dependent diabetes mellitus. Recent antecedent hypoglycemia reduces autonomic responses to, symptoms of, and defense against subsequent hypoglycemia. J Clin Inv 1993;91:819-928.

9. Matyka K, Evans M, Lomas J, et al. Altered hierarchy of protective responses against severe hypoglycemia in normal aging inhealthy men. Diabetes Care 1997;20:135-41.

10. Shorr RI, Ray WA, Daugherty JR, Griffin MR. Incidence and risk factors for serious hypoglycemia in older persons using insulin or sulfonylureas. Arch Intern Med 1997; 157:1681-6.

11. Seaquist ER, Anderson J, Childs B, et al. Hypoglycemia and diabetes: a report of a workgroup of the American Diabetes Association and the Endocrine Society. Diabetes Care 2013;36:1384-95.

12. Cook CB, Kongable GL, Potter DJ, et al. Inpatient glucose control: a glycemic survey of 126 U.S. hospitals. J Hospital Med 2009;4:E7-14.

13. Wexler DJ, Meigs JB, Cagliero E, et al. Prevalence of hyper- and hypoglycemia among inpatients with diabetes: a national survey of 44 U.S. hospitals. Diabetes Care 2007;30:367-9.

14. Turchin A, Matheny ME, Shubina M, et al. Hypoglycemia and clinical outcomes in patients with diabetes hospitalized in the general ward. Diabetes Care 2009;32:1153-7.

15. Swanson CM, Potter DJ, Kongable GL, et al. Update on inpatient glycemic control in hospitals in the United States. Endocr Pract 2011;17:853-61.
16. Brunkhorst FM, Engel C, Bloos F, et al. German Competence Network S. Intensive insulin therapy and pentastarch resuscitation in severe sepsis. New Engl J Med 2008;358:125-39.

17. Boucai L, Southern WN, Zonszein J. Hypoglycemia-associated mortality is not drug-associated but linked to comorbidities. Amer J Med 2011;124:1028-35.

18. Krinsley JS, Grover A. Severe hypoglycemia in critically ill patients: risk factors and outcomes. Crit Care Med 2007;35:2262-7.

19. Smith WD, Winterstein AG, Johns T, et al. Causes of hyperglycemia and hypoglycemia in adult inpatients. Am J Health Syst Pharm 2005;62:714-9.

20. Van der Crabben SN, Blumer RM, Stegenga ME, et al. Early endotoxemia increases peripheral and hepatic insulinsensitivity in healthy humans. J Clin Endocrinol Metab 2009;94:463-8.

21. Cryer PE, Davis SN, Shamoon H. Hypoglycemia in diabetes. Diabetes Care 2003;26:1902-12.

22. Vaghese P, Gleason V, Sorokin R, et al. Hypoglycemia in hospitalized patients treated with antihyperglycemic agents. J Hosp Med 2007;2:234-40.

23. Elliott MB, Schafers SJ, McGill JB, et al. Prediction and prevention of treatment-related inpatient hypoglycemia. J Diabetes Sci Technol 2012;6:302-9.

24. Cryer PE. Mechanisms of hypoglycemia-associated autonomic failure in diabetes. N Engl J Med 2013;369:362-72.

25. Cobaugh DJ, Maynard G, Cooper L, et al. Enhancing insulin-use safety in hospitals: practical recommendations from an ASHP foundation expert consensus panel. Am J Health Syst Pharm 2013;70:e18-e27.

26. Rubin DJ, Rybin D, Doros G, et al. Weight-based, insulin dose-related hypoglycemia in hospitalized patients with diabetes. Diabetes Care 2011;34:1723-8.

27. Curll M, DiNardo M, Noschese M, et al. Menu selection, glycaemic control, and satisfaction with standard and patient-controlled consistent carbohydrate diet meal plans in hospitalised patients with diabetes. Qual Saf Health Care 2010;19:355-9.

28. Korytkowski MT, Salata RJ, Koerbel GL, et al. Insulin therapy and glycemic control in hospitalized patients with diabetes during enteral nutrition therapy: a randomized controlled clinical trial. Diabetes Care 2009;32:594-6.

29. Donihi AC, Abriola C, Hall R, et al. Getting the timing right in the hospital: synching insulin administration with meal tray arrival. Diabetes 2010;59:1028-P.

30. Borzì V. Il controllo dell'iperglicemia nel paziente ospedalizzato non-critico : stop alla sliding scale! Il Giornale AMD 2013;16:287-93.

31. Donihi AC, DiNardo MM, DeVita MA, et al. Use of a standardized protocol to decrease medication errors and adverse events related to sliding scale insulin. Qual Saf Health Care 2006;15:89-91.

32. Gogitidze Joy N, Hedrington MS, Briscoe VJ, et al. Effects of acute hypoglycemia on inflammatory and proatherothrombotic biomarkers in individuals with type 1 diabetes and healthy individuals. Diabetes Care 2010; 33:1529-35.

33. Wright RJ, Newby DE, Stirling D, et al. Effects of acute insulin-induced hypoglycemia on indices of inflammation: putative mechanism for aggravating vascular disease in diabetes. Diabetes Care 2010;33:1591-7.

34. Fisher BM, Hepburn DA, Smith JG, Frier BM. Respon- 
ses of peripheral blood cells to acute insulin-induced hypoglycaemia in humans: effect of alpha-adrenergic blockade. Horm Metab Res Suppl Ser 1992;26:109-10.

35. Desouza C, Salazar H, Cheong B, et al. Association of hypoglycemia and cardiac ischemia: A study based on continuous monitoring. Diabetes Care 2003;26:1485-9.

36. Nordin C. The case for hypoglycaemia as a proarrhythmic event: basic and clinical evidence. Diabetologia 2010;53:1552-61.

37. Chow E, Bernjak A, Williams S, et al. Risk of cardiac arrhythmias during hypoglycemia in patients with type 2 diabetes and cardiovascular risk. Diabetes 2014;63: 1738-47.

38. Pop-Busui R. Cardiac autonomic neuropathy in diabetes: a clinical perspective. Diabetes Care 2010;33:434-41.

39. NICE-SUGAR Study Investigators; Finfer S, Liu B, et al. Hypoglycemia and risk of death in critically ill patients. New Engl J Med 2012;367:1108-18.

40. Malmberg K. Prospective randomised study of intensive insulin treatment on long term survival after acute myocardial infarction in patients with diabetes mellitus. DIGAMI (diabetes mellitus, insulin glucose infusion in acute myocardial infarction) study group. BMJ 1997; 314:1512-5.

41. Gruden G, Barutta F, Chaturvedi N, et al. Severe hypoglycemia and cardiovascular disease incidence in type 1 diabetes: the EURODIAB Prospective Complications Trial. Diabetes Care 2012;35:1598-604.

42. Rajendran R, Rayman G. Serious harm from inpatient hypoglycaemia: a survey of hospitals in the UK. Diabetic Medicine 2014 [In press]

43. Kahn KJ, Myers RE. Insulin-induced hypoglaecyemia in the nonhuman primate. I. Clinical consequences Clin Dev Med 1971;39/40:185-94.

44. The Diabetes Control and Complications Trial/Epidemiology of Diabetes Interventions and Complications (DCCT/EDIC) StudyResearch Group. Long-term effects of diabetes and its treatment on cognitive function. N Engl J Med 2007;356:1842-52.

45. Garg R, Hurwitz S, Turchin A, et al. Hypoglycemia, with or without insulin therapy, is associated with increased mortality among hospitalized patients. Diabetes Care 2013;36:1107-10.

46. Bagshaw SM, Egi M, George C, Bellomo R. Early blood glucose control and mortality in critically ill patients in Australia. Crit Care Med 2009;37:463-70.

47. Kosiborod M, Inzucchi SE, Goyal A, et al. Relationship between spontaneous and iatrogenic hypoglycemia and mortality in patients hospitalized with acute myocardial infarction. JAMA 2009;301:1556-64.

48. Curkendall SM, Natoli JI, Alexander CM, et al. Economic and clinical impact of inpatient Diabetic hypoglycemia. Endocr Pract 2009;15:302-12.

49. Mendez CE, Tanenberg RJ, Mok K, et al. Increased glycemic variability is independently associated with lengthof stay and mortality in noncritically ill hospitalized patients. Diabetes Care 2013;36:4091-7.

50. Pietras SM, Hanrahan P, Arnold LM, et al. State of the art inpatient diabetes care: the evolution of an academic hospital. Endocr Pract 2010;16:512-21.

51. Baldwin D, Zander J, Munoz C, et al. A randomized trial of two weight-based doses of insulin glargine and glulisine in hospitalized subjects with type 2 diabetes and renal insufficiency. Diabetes Care 2012;35:1970-4.
52. Brunner GA, Hirschberger S, Sendlhofer G, et al. Postprandial administration of the insulin analogue insulin aspart in patients with type 1 diabetes mellitus. Diabetic Medicine 2000;17:371-5.

53. Hawkins K, Korytkowski M. Diabetes in specific settings: inpatient management and special procedures. Alexandria, VA: American Diabetes Association; 2013.

54. Home PD, Lindholm A, Hylleberg B, et al. Improved glycemic control with insulin aspart: a multicenter randomized double-blind crossover trial in type 1 diabetic patients. Diabetes Care 1998;21:1904-9.

55. Anderson Jr JH, Brunelle RL, Koivisto VA, et al. Reduction of postprandial hyperglycemia and frequency of hypoglycemia in IDDM patients on insulin-analog treatment. Multicenter Insulin Lispro Study Group. Diabetes 1997;46:265-70

56. Guerra YS, Lacuesta EA, Yrastorza R, et al. Insulin injections in relation to meals in the hospital medicine ward: comparison of 2 protocols. Endocr Pract 2011;17:737-46.

57. Pedersen-Bjergaard U, Kristensen PL, Beck Nielsen H, et al. Effect of insulin analogues on risk of severe hypoglycaemia in patients with type 1 diabetes prone to recurrent severe hypoglycaemia (HypoAna trial): a prospective, randomised, open-label, blinded-endpoint crossover trial. Lancet Diabetes Endocrinol 2014;7:553-65.

58. Heller SR, Amiel SA, Mansell P. Effect of the fast-acting insulin analog lispro on the risk of nocturnal hypoglycemia during intensified insulin therapy. U.K. Lispro Study Group. Diabetes Care 1999;22:1607-11.

59. Heller S, Colagiuri S, Vaaler S, et al. Reduced hypoglycemia with insulin aspart: a double-blind, randomized crossover trial in type 1 diabetic patients. Diabetes 2001;50:A137.

60. Rosenstock J, Dailey G, Massi-Benedetti M, et al. Reduced hypoglycemia risk with insulin glargine: a metaanalysis comparing insulin glargine with human NPH insulin in type 2 diabetes. Diabetes Care 2005;28:950-7.

61. Haak T, Tiengo A, Draeger E, et al. Lower withinsubject variability of fasting blood glucose and reduced weight gain with insulin detemir compared to NPH insulin in patients with type 2 diabetes. Diabetes Obes Metab 2005;7:56-64.

62. Heise T, Pieber TR. Towards peakless, reproducible and long-acting insulins. An assessment of the basal analogues based on isoglycaemic clamp studies. Diabetes Obes Metab 2007;9:648-59.

63. Rodbard HW, Jellinger PS, Davidson JA, et al. Statement by an American Association of Clinical Endocrinologists/American College of Endocrinology consensus panel on type 2 diabetes mellitus: an algorithm for glycemic control. Endocr Pract 2009;15:540-59.

64. Borzì V, Frasson S, Solerte B, et al. Hypoglycemia in type 2 diabetic patients hospitalized in Internal Medicine: which predictors and outcomes? Results of the DIAMOND study from the Scientific Society FADOI. European and Swiss Congress of Internal Medicine 2014; abstract 199.

65. Heise T, Hermanski L, Nosek L, et al. Insulin degludec: four times lower pharmacodynamic variability than insulin glargine under steady-state conditions in type 1 diabetes. Diabetes Obesity Metabo 2012;14:859-64.

66. Heller S, Buse J, Fisher M, et al. Insulin degludec, an ultra-longacting basal insulin, versus insulin glargine in 
basal-bolus treatment with mealtime insulin aspart in type 1 diabetes (BEGIN Basal-Bolus Type 1): a phase 3 , randomised, open-label, treat-to-target non-inferiority trial. Lancet 2012;379: 1489-97.

67. Garber AJ, King AB, Del Prato S, et al. Insulin degludec, an ultralongacting basal insulin, versus insulin glargine in basal-bolus treatment with mealtime insulin aspart in type 2 diabetes (BEGIN Basal-Bolus Type 2): a phase 3 , randomised, open-label, treat-to-target non-inferiority trial. Lancet 2012;379:1498-507.

68. Sorli C, Warren M, Oyer D, et al. Elderly patients with diabetes experience a lower rate of nocturnal hypoglycaemia with insulin degludec than with insulin glargine: a meta-analysis of phase IIIa trials. Drugs Aging 2013; 30:1009-18.

69. Meneghini L, Bain S, Atkin SL, et al. The efficacy and safety of insulin degludec given in variable once-daily dosing intervals compared with insulin glargine and insulin degludec dosed at the same time daily. Diabetes Care 2013;36:858-64.

70. Gulli G, Frasson S, Borzì V, et al. Effectiveness of an educational intervention on the management of type 2 diabetic patients hospitalized in internal medicine: results from the FADOI-DIAMOND study. Acta Diabetologica 2014;51:765-70. 